\title{
Serious Right Coronary Artery Thrombosis Revealing Behçet's Disease in a Female Patient: A Case Report
}

\author{
Fadoua Mouedder ${ }^{1,2}$, Karima Benbouchta ${ }^{1,2}$, Nabila Ismaili ${ }^{1,3}$, Noha Elouafi ${ }^{1,3}$ \\ 1. Cardiology, Mohammed VI University Hospital, Oujda, MAR 2. Cardiology, Mohammed I University, Epidemiological \\ Laboratory of Clinical Research and Public Health, Oujda, MAR 3. Cardiology, Mohammed I University, Oujda, MAR
}

Corresponding author: Fadoua Mouedder, fadoua.mouedder@gmail.com

\begin{abstract}
Although atherosclerosis remains the major cause of acute coronary syndrome, there are many other etiologies that should be taken into account, especially in young patients with no atherosclerotic risk factors. Coronary involvement is extremely rare in patients with Behçet's disease, notably in young patients. In addition, acute inferior myocardial infarction revealing Behçet's disease has rarely been reported. Through this article, we report a case of Behçet's disease with arterial involvement diagnosed after myocardial infarction resulting from thrombosis of the right coronary artery in a 50-year-old woman with no specific medical history.
\end{abstract}

Review began 10/02/2020 Review ended 10/24/2020 Published 11/08/2020

\section{(c) Copyright 2020}

Mouedder et al. This is an open access article distributed under the terms of the Creative Commons Attribution License CC-BY 4.0., which permits unrestricted use, distribution, and reproduction in any medium, provided the original author and source are credited.
Categories: Cardiology, Internal Medicine

Keywords: st-segment elevation, right coronary artery, coronary thrombosis, behçet disease, cardiac involvement

\section{Introduction}

Behçet's disease (BD) is a systemic vasculitis of unknown etiology, characterized by remitting and relapsing episodes of genital and oral ulcers, ocular lesions, and a number of systemic manifestations [1]. Vascular involvement in BD can involve both veins and arteries of any diameter. Venous involvement in BD is more frequent than arterial involvement; the latter can be life-threatening and mainly concerns large arteries but also can affect the peripheral arteries. Coronary artery disease is very rare in BD and poses several challenges. We report a female patient diagnosed with BD who presented with acute coronary syndrome caused by right coronary artery thrombosis.

\section{Case Presentation}

We report the case of a 50-year-old woman with a four-year history of bronchiectasis treated with salbutamol, with no known major risk factors for atherosclerosis causation. This patient presented to the emergency department with acute and constrictive chest pain evolving for the last six hours. Physical examination noted stable hemodynamic status without signs of heart failure. ECG showed ST-segment elevation in inferior leads (Figure 1).

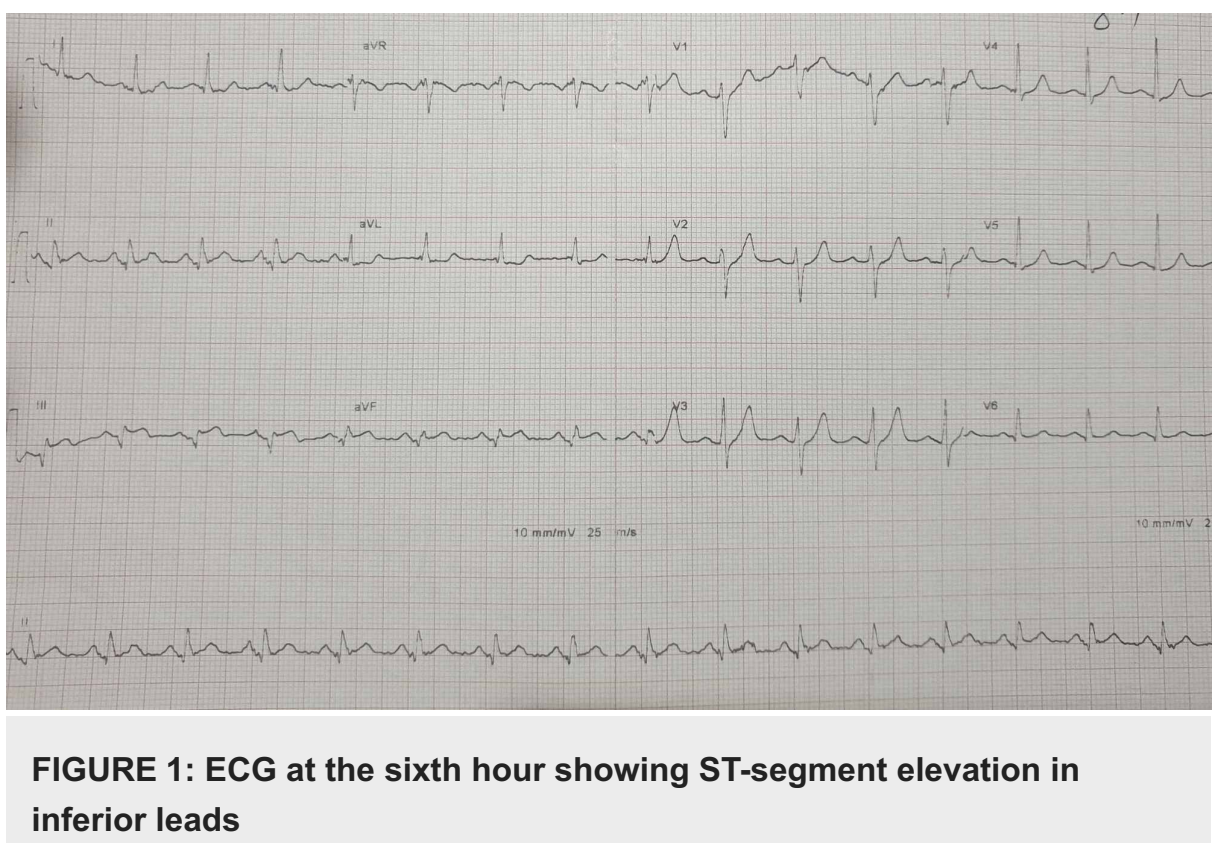




\section{Cureus}

The diagnosis of a non-complicated inferior ST-segment elevation myocardial infarction (STEMI) seen at the sixth hour was made. Given the resolution of chest pain, the patient did not benefit from

thrombolytic therapy. Given the fact that primary angioplasty was not available at that hour, treatment with antithrombotics was started combining aspirin, clopidogrel, and enoxaparin. Echocardiography revealed extended akinesis of the inferior and inferoseptal walls and hypokinesia of the inferolateral wall, without intraventricular thrombus. Left ventricle ejection fraction was estimated about $45 \%$ without mechanical complications. At the tenth hour, the patient underwent coronary angiography, which showed a fresh thrombus floating in the distal part of the right coronary artery with thrombolysis in myocardial infarction (TIMI) 0 flow and angiographically normal left coronary system. Thrombus aspiration using a catheter was performed, retrieving red thrombi with the restoration of TIMI 3 flow without placement of a stent (Figure 2).
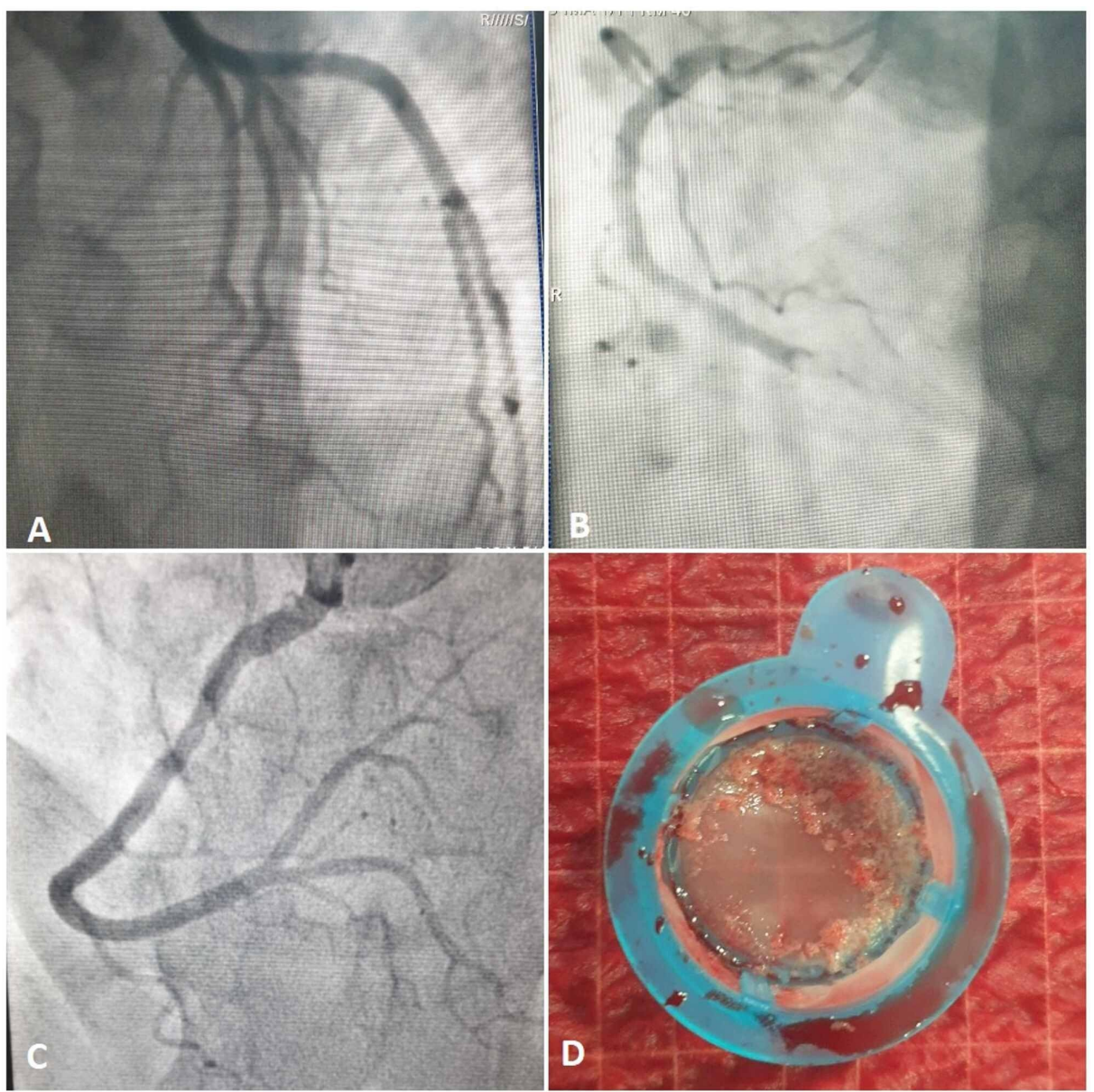

\section{FIGURE 2: Coronary angiography at the acute phase of myocardial} infarction

a: Angiographically normal left coronary system

b: Distal right coronary artery thrombosis with TIMI 0 flow

c, d: Thrombus aspiration retrieving red thrombi restoring TIMI 3 flow

TIMI - thrombolysis in myocardial infarction

The patient was kept on antiglycoprotein IIb-IIIa (anti-GpIIb-IIIa) for 24 hours.

The etiological investigation did not reveal other atherothrombosis risk factors. Given the important thrombotic burden in the right coronary artery associated with angiographically normal coronary arteries, thrombophilia was highly suspected. Screening for anticardiolipin antibodies, hyperhomocysteinemia, antithrombin, and protein $\mathrm{S}$ and $\mathrm{C}$ deficiencies were negative. After careful questioning, the patient revealed a history of arthralgias and recurrent buccal and genital ulcers. Physical examination revealed a buccal ulcer 


\section{Cureus}

and a vaginal scar that would be compatible with a vaginal ulcer sequel, which were highly evocative of BD. Fundoscopy showed signs of uveitis. Fluorescein angiography showed signs of vasculitis and microaneurysms suggestive of BD (Figure 3).
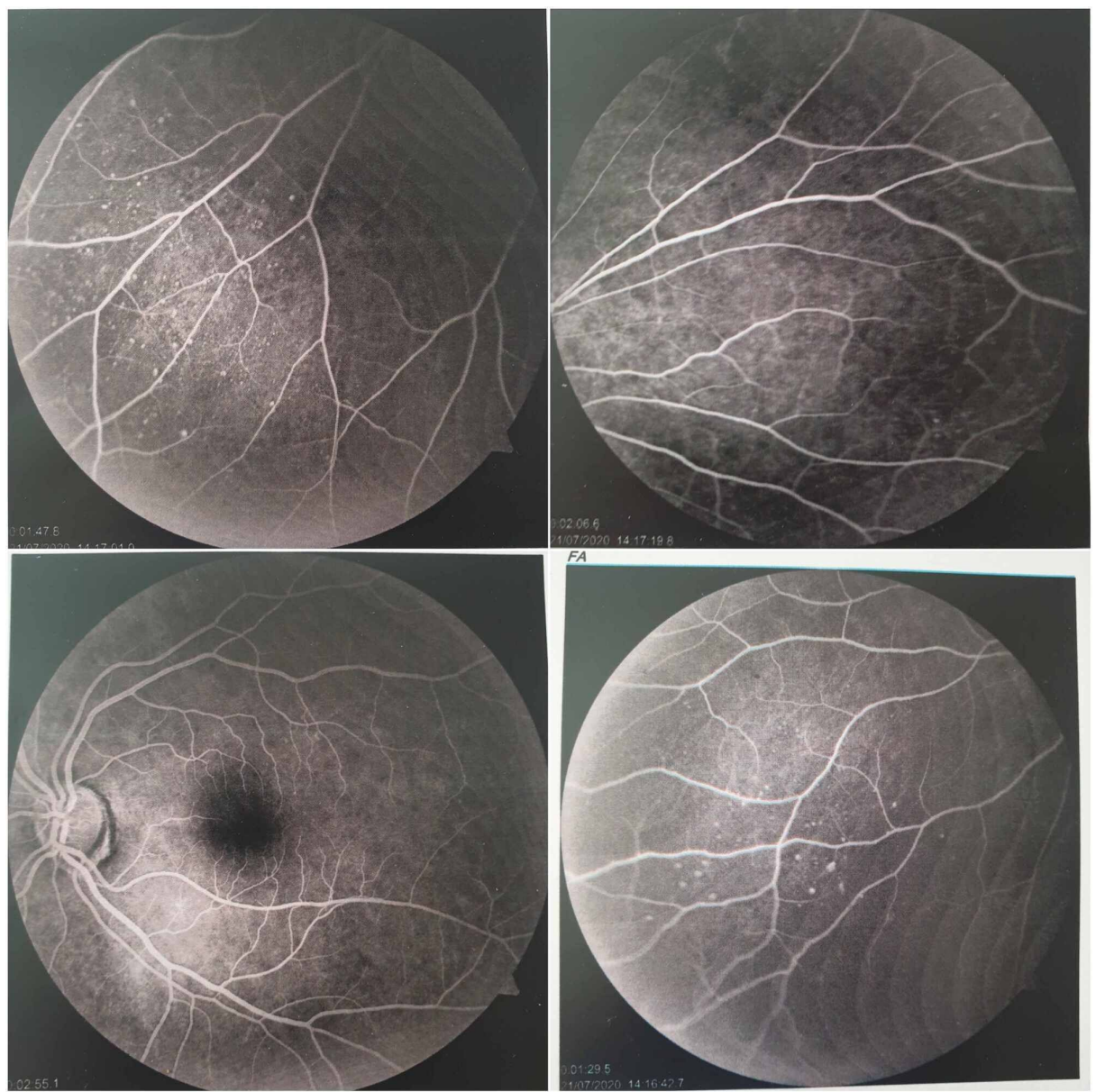

FIGURE 3: Fluorescein angiography showing signs of vasculitis and microaneurysms suggestive of Behçet's disease

Based on the international criteria for $\mathrm{BD}$, the diagnosis of angio-Behçet was retained. As a consequence, the patient received dual antiplatelet therapy (acetylsalicylate and clopidogrel), antivitamin K (Sintrom ${ }^{\circledR}$ ), bisoprolol, ramipril, and atorvastatin. The clinical course remained uneventful, and echocardiography repeated a month later showed improved left ventricular ejection fraction at 55\% (Figure 4).

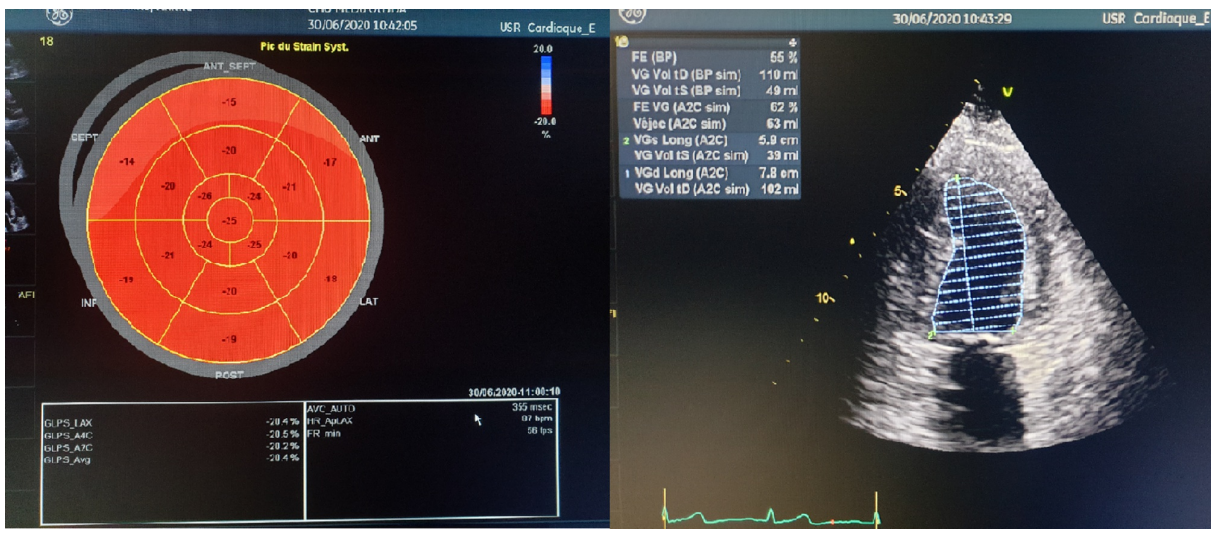

FIGURE 4: Echocardiography showing recovery of normal LVEF

LVEF - left ventricular ejection fraction 


\section{Discussion}

$\mathrm{BD}$ is a systemic vasculitis of unknown etiology. It is more common in young adults from the Middle East and Mediterranean rim and affects twice as many men as women. Its diagnosis is clinical, based on international criteria that have high sensitivity [1,2].

BD is characterized by remitting and relapsing episodes of oral ulcers, genital ulcers, skin, and ocular lesions. Many systems may be involved, such as neurological, musculoskeletal, and cardiovascular systems [3]. Cardiovascular involvement is rare but can have different manifestations and can be life-threatening. According to several studies, the prevalence of cardiovascular involvement is around 29\% [4].

Valvular disorder, intraventricular thrombosis, and coronary arteries aneurysms are the most reported cardiac manifestations in $\mathrm{BD}$, but there are other disorders such as myocarditis, pericarditis, endocarditis, and conductive tissue disorders [5]

The vascular tropism of BD is manifested by phlebitis, which is a major sign of the disease [6]. Arterial involvement is rare compared to venous involvement. Arterial involvement makes up only $20 \%$ of vascular complications [7]. Arteries of any size may be affected resulting from either occlusion or an aneurysm. The major involved arteries are pulmonary, femoral, popliteal, subclavian, and carotid arteries. Coronary involvement is extremely rare, especially in young patients [8].

The majority of cases described in the literature, which presented with coronary events, were previously diagnosed with $\mathrm{BD}$, and followed regular treatment. Less frequently, coronary complications can occur as the first manifestation of this disease, which was our patient's case.

Coronary artery disease is very rare in BD but poses several diagnostic and therapeutic challenges and can be life-threatening. To our knowledge, there are three forms of coronary artery involvement during Behcet's disease: pseudoaneurysms, stenosis, and thrombosis [9].

The exact cause of acute coronary syndrome in patients with BD is not clearly understood. Unlike other inflammatory diseases such as systemic lupus erythematosus, BD is not associated with the acceleration of atherosclerosis $[10,11]$. Thus, the main mechanisms involved are stenotic lesions and coronary aneurysms, which are the most frequently detected lesions. Also, coronary vasculitis can lead to coronary occlusion by causing intimal fibrous thickening [10]. The compression of the coronary artery by external aneurysmal dilation of the left sinus of Valsalva and also impaired microvascular function are thought to be causes of acute coronary events in this context [12]. The presence of a fresh floating thrombus in angiographically normal coronary arteries has also been reported in the literature suggesting an underlying pro-thrombotic etiology [8].

During BD, two mechanisms can explain thrombosis: hypercoagulable state and vasculitis. The hypercoagulable state is believed to be due to increased platelet aggregation but also to inhibition of fibrinolysis. Increased platelet aggregation is explained by endothelial dysfunction, leading to decreased prostacyclin levels and increased production of von Willebrand factor and factor VIII [13, 14]. Vasculitis of $\mathrm{BD}$ affects all layers of vessels; during the acute phase, it is characterized by the presence of lymphocytic infiltrate and significant fibrous at an advanced stage. Vasculitis remains the main mechanism of thrombosis [9]. For our patient, both vasculitis and hypercoagulable state appeared to be involved in coronary thrombosis.

In the literature, several therapeutic approaches have been defined for managing myocardial infarction (MI) in patients with BD. According to the European Society of Cardiology (ESC) guidelines for the management of acute myocardial infraction in a patient with ST-segment elevation, reperfusion therapy is indicated in all patients within 12 hours of symptom onset and persistent ST-segment elevation, and primary percutaneous coronary intervention (PCI) is the recommended reperfusion strategy over fibrinolysis if performed within 120 minutes of the first medical contact [15]. That's why some authors suggested that percutaneous coronary dilatation was the most recognized revascularization option during STEMI secondary to BD's coronary thrombosis [16]. Cetin et al. proposed that thrombus aspiration and administration of GPII b III a inhibitors should be used first, ballooning and/or stenting should be selected as the second choice on account of pathergy like effect [17]. But in some cases treating a coronary thrombosis in patients with BD with PCI tends to result in an increased rate of stent thrombosis and thrombosis recurrence during longterm follow-up and carries a high possibility for the development of coronary artery aneurysm [18]. Therefore, Tsuboi et al. recommended that it is important to administer immunosuppressive drugs, in addition to antithrombotic drugs, such as warfarin, before and after PCI in vasculo-BD to prevent relapse or exacerbation. Corticosteroids, colchicine, azathioprine, and cyclophosphamide were the most commonly used agents in previous reports [19]. Alternatively, another approach is the administration of thrombolytic therapy at the early hours of MI. Kosar et al. reported a successful case of fibrinolysis in a patient with BD [20]. 
Some authors report using only a high dose of corticosteroids to treat some cases of MI in patients with BD. Hattori and Kawana reported a case of a patient treated using sodium methylprednisolone succinate at a dose of $1000 \mathrm{mg}$ for three days, followed by oral administration of prednisolone at a dose of $60 \mathrm{mg} / \mathrm{day}$, leading to improvement of all the symptoms after three weeks and normalization of the electrocardiogram findings [18]. Finally, the therapeutic approach remains complex and varies as to the underlying pathology.

In our case, the initial sole antiaggregant, anticoagulant treatments, and thrombus aspiration contributed to the recanalization of coronary thrombosis and the improvement of cardiac ischemia. The patient also received dual antiplatelet therapy (acetylsalicylate and clopidogrel), antivitamin K (Sintrom), bisoprolol, ramipril, and atorvastatin. As the diagnosis of Behcet's disease was only suspected, we chose to start a treatment combining corticosteroids and immunosuppressants after establishing the diagnosis according to international criteria. The clinical course was good, the patient did not develop heart failure signs, and repeated echocardiography showed improved left ventricular ejection fraction at $55 \%$.

\section{Conclusions}

Although coronary artery thrombosis, as a cardiac involvement, is a rare manifestation of BD, but may worsen the prognosis of this disease and should be kept in mind, especially in young patients presenting with chest pain accompanied by systemic involvement. The therapeutic approach for this complication remains complex. More research is needed for a better understanding and management of this complication.

\section{Additional Information \\ Disclosures}

Human subjects: Consent was obtained by all participants in this study. Conflicts of interest: In compliance with the ICMJE uniform disclosure form, all authors declare the following: Payment/services info: All authors have declared that no financial support was received from any organization for the submitted work. Financial relationships: All authors have declared that they have no financial relationships at present or within the previous three years with any organizations that might have an interest in the submitted work. Other relationships: All authors have declared that there are no other relationships or activities that could appear to have influenced the submitted work.

\section{References}

1. International Study Group for Behçet's Disease: Criteria for diagnosis of Behçet's disease . Lancet. 1990, 335:1078-1080. 10.1016/0140-6736(90)92643-V

2. B'chir Hamzaoui S, Harmel A, Bouslama K, et al.: Behçet's disease in Tunisia. Clinical study of 519 cases . Rev Med Interne. 2006, 27:742-750. 10.1016/j.revmed.2006.07.019

3. Mendes D, Correia M, Barbedo M, et al.: Behçet's disease-a contemporary review . J Autoimmun. 2009, 32:178-188. 10.1016/j.jaut.2009.02.011

4. Gurler A, Boyvat A, Tursen U: Clinical manifestations of Behcet's disease: an analysis of 2147 patients Yonsei Med J. 1997, 38:423-427. 10.3349/ymj.1997.38.6.423

5. Marzban M, Mandegar MH, Karimi A, et al.: Cardiac and great vessel involvement in "Behcet's disease" . J Card Surg. 2008, 23:765-768. 10.1111/j.1540-8191.2008.00607.x

6. Wechsler B, Lê Thi Huong Du LT, de Gennes C, et al.: Arterial manifestations of Behçet's disease: report of 12 cases. Rev Med Interne. 1989, 10:303-311. 10.1016/s0248-8663(89)80027-x

7. Düzgün N, Ateş A, Aydintuğ OT, Demir O, Olmez U: Characteristics of vascular involvement in Behçet's disease. Scand J Rheumatol. 2006, 35:65-68. 10.1080/03009740500255761

8. Ayari J, Mourali MS, Farhati A, Mechmeche R: Left main coronary artery thrombosis revealing angio-Behçet syndrome. Egypt J Intern Med. 2014, 26:88-90. 10.4103/1110-7782.139578

9. Ko GY, Byun JY, Choi BG, Cho SH: The vascular manifestations of Behçet's disease: angiographic and CT findings. Brit J Radiol. 2000, 73:1270-1274. 10.1259/bjr.73.876.11205670

10. Seyahi E, Uğurlu S, Cumalı R, et al.: Atherosclerosis in Behçet’s disease syndrome. Semin Arthritis Rheum. 2008, 38:1-12. 10.1016/j.semarthrit.2007.09.009

11. Gasparyan AY, Stavropoulos-Kalinoglou A, Mikhailidis DP, et al.: The rationale for comparative studies of accelerated atherosclerosis in rheumatic diseases. Curr Vasc Pharmacol. 2010, 8:437-449. 10.2174/157016110791330852

12. Lee S, Lee CY, Yoo KJ: Acute myocardial infarction due to an unruptured sinus of valsalva aneurysm in a patient with Behçet's syndrome. Yonsei Med J. 2007, 48:883-885. 10.3349/ymj.2007.48.5.883

13. Özoran K, Düzgun N, Gürler A, Tutkak H, Tokgöz G: Plasma von Willebrand factor, tissue plasminogen activator, plasminogen activator inhibitor, and antithrombin III levels in Behcet's disease. Scand J Rheumatol. 1995, 24:376-382. 10.3109/03009749509095184

14. Hutchison SJ, Belch JJ: Behçet's syndrome presenting as myocardial infarction with impaired blood fibrinolysis. Heart. 1984, 52:686-687. 10.1136/hrt.52.6.686

15. Ibanez B, James S, Agewall S, et al.: 2017 ESC Guidelines for the management of acute myocardial infarction in patients presenting with ST-segment elevation: The Task Force for the management of acute myocardial infarction in patients presenting with ST-segment elevation of the European Society of Cardiology (ESC). Eur Heart J. 2018, 39:119-177. 10.1093/eurheartj/ehx393

16. Drobinski G, Wechsler B, Paviej A, et al.: Emergency percutaneous coronary dilatation for acute myocardial infarction in Behçet's disease. Eur Heart J. 1987, 8:1133-1136. 10.1093/oxfordjournals.eurheartj.a062179

17. Cetin M, Ozturk U, Cakici M, Yildiz E: Acute inferior myocardial infarction in a young male patient 


\section{Cureus}

associated with Behcet's disease and sildenafil. BMJ Case Rep. 2014, 2014: 10.1136/bcr-2013-201189

18. Hattori S, Kawana S: Behçet's syndrome associated with acute myocardial infarction. J Nippon Med Sch. 2003, 70:49-52. 10.1272/jnms.70.49

19. Tsuboi K, Tamura M, Sone N, et al.: Recurrent myocardial infarction associated with vasculo-Behçet's disease: a case report. Mod Rheumatol. 2020, 4:Mod Rheumatol . 10.1080/24725625.2019.1685149

20. Kosar F, Sahin I, Gullu H, Cehreli S: Acute myocardial infarction with normal coronary arteries in a young man with the Behcet's disease. Int J Cardiol. 2005, 99:355-357. 10.1016/j.ijcard.2003.11.039 